\title{
How to develop a niche: Focus on adult cardiac surgery
}

\author{
Ahmet Kilic, MD

\footnotetext{
From the Division of Cardiac Surgery, Department of Surgery, Wexner Medical Center, The Ohio State University, Columbus, Ohio.

Disclosures: Author has nothing to disclose with regard to commercial support.

Received for publication June 30, 2015; revisions received Aug 3, 2015; accepted for publication Sept 4, 2015; available ahead of print Oct 15, 2015.

Address for reprints: Ahmet Kilic, MD, Division of Cardiac Surgery, Department of Surgery, The Ohio State University, Wexner Medical Center, 410 W 10th Ave, N-816 Doan Hall, Columbus, OH 43210 (E-mail: Ahmet.Kilic@osumc.edu).

J Thorac Cardiovasc Surg 2016;151:636-

0022-5223/\$36.00

Copyright $(\subset 2016$ by The American Association for Thoracic Surgery

http://dx.doi.org/10.1016/j.jtcvs.2015.09.029
}

The switch from surgical trainee to starting your practice, and for all purposes, your career, is often the most difficult transition in adult cardiac surgery. In contrast to other transitions, this one is a time with no defined blueprint and no other trainee with whom to share and commiserate about the highs and lows that you will encounter within the initial, pivotal years. In preparation for this important journey, having a clear idea of your expectations, capabilities, and limitations is imperative. In addition, institutional expectations, needs, available resources, and hospital commitments should all be factored into the career path decision. The marriage of institutional and personal desires should allow one to set both short- and long-term goals, with the ultimate goal of being recognized as a "go-to" surgeon in a particular niche.

\section{IDENTIFYING A NICHE}

A host of factors are relevant to identifying a niche, to begin to define your career. The success of this process depends on a symbiotic relationship with your institution, regardless of whether it is an academically oriented university or a nonacademic hospital. Currently, the major areas of growth and need in cardiac surgery are: (1) complex aortic surgery including endovascular techniques; (2) minimally invasive valve procedures, including robotic and transcatheter approaches; (3) thoracoscopic and standalone anti-arrhythmia surgeries; and (4) end-stage heart failure therapies, including transplantation, ventricular assist devices, and extracorporeal membrane oxygenator management.

\section{WHAT ARE YOU PASSIONATE ABOUT?}

Perhaps the first and most important question is to identify an area of adult cardiac surgery that you enjoy, and for which you have passion. This area should be one that is readily evident to you and those around you (hint: It is the thing you find least annoying when you are called in on weekends, nights, and holidays). To maximize your chances of success in this area, practice "knowledge aggregation," ie, research as much as you can about the state of the field, its current shortcomings, and future

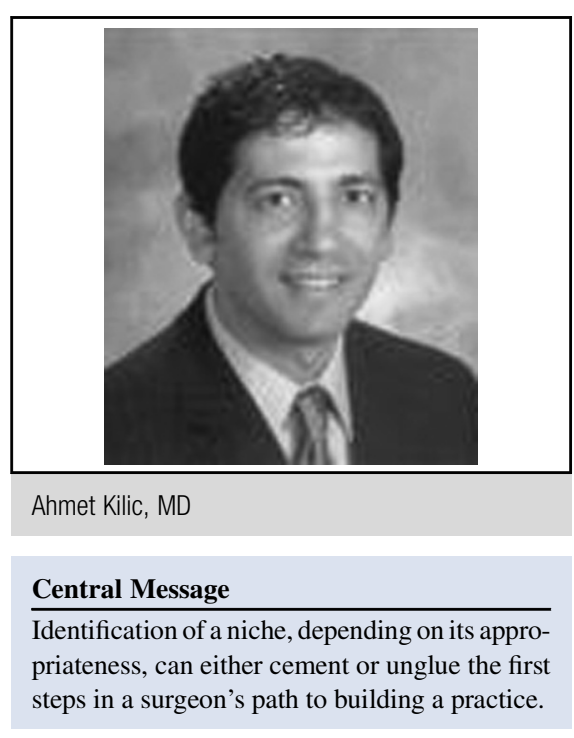

See Editorial Commentary page 640.

directions. A continued, focused immersion through reading, conference attendance, and interactions with the current, authoritative leaders in the field will give you added credibility and immediate traction.

\section{ARE YOU ADEQUATELY PREPARED?}

The next question is whether you are adequately trained to manage all aspects of the disease process involved in the chosen area. For some areas, traditional cardiac surgery training will suffice, but for others, you will need advanced training. The time period before starting your career path should be all about "experience aggregation." Whether through more-specific training, completion of a specialized fellowship, an externship at a high-volume center, or double scrubbing on appropriate cases, the goal of the final months of training should be to establish competency, proficiency, and mastery of a particular technique.

The decision to pursue a specific niche usually occurs during training; therefore, focusing your energies during the final parts of your training is imperative. During this time, being a little selfish is acceptable, to ensure that you are getting what you need to be successful. Some creativity may be required in asking for elective time to get more experience in certain types of cases. Alternatively, externships at outside institutions known for dealing with your proposed niche, and travel time to attend meetings with experts in the field, are helpful in accumulating niche-related experience as well.

In certain situations, whether because of the training environment or the complexity of the specialty, seeking additional training will be necessary. The purpose of this article 
is not to debate 2- versus 3-year training programs, nor the need for "extra fellowship" training versus traditional residency programs. However, having stated an interest in a particular niche, a very uncomfortable position is to find that you lack the prerequisite training, exposure, and/or case volumes needed. If the answer to the question "Are you adequately prepared?" is "no," then additional training is appropriate. In addition to the methods just described, partnerships and grants from academic organizations and/or industry can be sought out for further exposure.

For a minority of trainees, a predefined niche is set by a commitment to a specific hospital, or geographic region, well in advance of the end of their training. For most trainees, this situation does not apply, and the decision to pursue a particular area of adult cardiac surgery is made before deciding on a particular employer. This approach does stress the importance of confirming that a specific need, at a specific institution, and at a specific time does exists. An objective assessment of each is vital, and an alignment of all 3 will ensure a successful early trajectory in your chosen career.

\section{IS THE ENVIRONMENT RIGHT FOR YOUR SUCCESS?}

After identifying what you are passionate about, and ensuring adequate attainment of a skillset, the next question is what kind of environment is needed to be successful. The assessment of need can be made by looking at a specific hospital and asking if the necessary personnel, institutional resources, and ancillary staff are present. Another major factor to consider is whether a real void exists, although a void to fill can be a negative or a positive, depending on the context. You want to be recruited to fill a void; however, filling a highly specialized area coming out of training, with no previous institutional experience or support structure is a gamble. You may have gained excellent surgical technique and clinical judgement during training, but no individual is an island, and the success of your program will be contingent on the team you assemble around you.

In contrast, if the institution is already known for your area of interest, you have to either play a supportive role initially or build on the existing expertise in your own way. This approach entails not only gaining the confidence of others in your being a clinically competent and capable surgeon, but also stepping out of the shadows of those that filled the role previously.

In some environments, caution should be exercised. You should be weary of going into the same city or region where a competing hospital has national dominance in your niche. In these situations, the surgical volume will be an issue, and you will undoubtedly be unfairly expected to compete with an experienced and renowned center. Another essential question to ask is whether others have been recruited to fill your position previously. You need to identify the barriers to their success, other contributing factors, and threats in the environment that made the relationship fail. Another situation to be aware of is the transition role. These are cases in which an institution has had success in a particular area, but has a surgeon void that they will fill only while in the market for an experienced surgeon.

The ideal context in which to build your practice is with a strong commitment from both the hospital and department. You need to ensure that your area of expertise is a priority not only to you, but also to all parties involved. Appropriate investments in personnel and financial resources are essential. A useful exercise is to put together a business proposal that includes a budget, key personnel, short- and long-term goals, "deliverables," as well as a cost-benefit analysis in ensuring a clear goal and destination.

\section{IMPORTANCE OF MENTORSHIP}

The transition from residency to practice, and the selection of an appropriate niche, are no easy tasks. Even though all of the aforementioned conditions may seem right to you, a critically important factor is to have guidance and input from a mentor in making career decisions. Mentorship can take many forms and should not be confined to only your niche, your specialty, or even your field. As in making a choice to pursue cardiothoracic surgery training, listening to valuable input from those who have experience and have your best interest at heart is important. A good mentor is someone who guides you not only through the technical aspects of surgery, but also through life as a cardiothoracic surgeon. ${ }^{1}$ Among the qualities of a good mentor-mentee relationship are trust, regular communication, and availability. The importance of mentorship in identifying a niche and transitioning to your career cannot be underestimated-it is recurring advice from those who have navigated the transition successfully. ${ }^{2}$

\section{DEFINE YOUR NICHE}

The niches to avoid are ones that you do not feel passionately about, in which volume is unlikely to grow, no resources are available, no consistent referral patterns exist, the region has too many threats, and no institutional support or structure is available. After identifying a niche by ruling out those that are unlikely to be successful, the next step is to define and develop yourself. This period is often one when you need to be selfish and start thinking like a business person, with the product being you. In the current environment, the top 4 areas in which to develop a niche are: aortic, valve, arrhythmia, and end-stage heart failure surgeries. Each one presents specific needs, and has skillset requirements and nuances that make it challenging and exciting in its own way.

\section{AORTIC SURGERY}

Undoubtedly, aortic surgery has changed with the advent of endovascular approaches, and to be a complete aortic surgeon, you have to be versatile and competent in both open and endovascular techniques. Although examples 
can be found of cardiac surgeons who are successful without completing any regimented endovascular training, most current aortic surgeons have finished a dedicated time of extra training, concentrating on aortic pathology. In the right environment, you can team up with an interventional radiologist, a vascular surgeon, or an interventional cardiologist versed in endovascular techniques, to build an aortic program, but this relationship would have to be symbiotic, with a common goal.

As with other niches, actively attending and participating in conferences, such as the American Association for Thoracic Surgery (AATS) Aortic Symposia, will allow you to interact with leaders in the field and learn the latest techniques and trends in aortic surgery. Similarly, being proficient with the data and lessons learned from the International Registry of Acute Aortic Dissection, and various techniques from the literature, will allow you to develop an aortic program and showcase a knowledge base at your home institution. In addition, a strong relationship with a geneticist and a basic scientist will allow you to develop an internal reputation in dealing with patients with bicuspid aortic disease, connective tissue disorders, and other familial vasculopathies.

\section{VALVE SURGERY}

Valve surgery, much like aortic surgery, has become less invasive and increasingly more specialized. Minimally invasive techniques, including robotic mitral valve repairs, mini-sternotomy and/or thoracotomy aortic valve replacements, and transcatheter aortic valve and mitral valve interventions, have all become specialized techniques in the cardiac surgeon's armamentarium. Admirably, transcatheter aortic valve replacement and the mitral valve clip have been developed in conjunction with interventional cardiologists, in a structural-heart-disease-team concept, and an excellent working relationship between the 2 is paramount in this niche. Most cardiothoracic training programs give some exposure to the transcatheter aortic valve replacement procedures, and the American Board of Thoracic Surgery now requires 20 interventional cases, but true mastery for percutaneous and robotic interventions requires further, specialized surgical training. ${ }^{3}$

A healthy knowledge of The Society of Thoracic Surgeons (STS)/American College of Cardiology (ACC) Transcatheter Valve Therapies Registry is a must. Attendance at the AATS Mitral Conclave, the AATS/ACC Heart Valve Summit, and/or the International Society for Minimally Invasive Cardiothoracic Surgery, is a good start in knowledge aggregation, as well as interaction with leaders in the field of valvular therapy. The successful mitral valve surgeon has a healthy arrhythmia practice as well, with the 2 areas being complementary. Other wire skills and valve repair techniques can be obtained through workshops like the STS University, or through more-formal, dedicated fellowship time with experts. Industry grants, such as the Intuitive Surgical
Clinical Robotics Fellowship, are other avenues to gather competency while obtaining salary support.

\section{ARRHYTHMIA SURGERY}

The incidence and awareness of atrial fibrillation continue to rise, as does the therapeutic interventions used in addressing both rhythm control and the left atrial appendage. The successful anti-arrhythmia surgeon needs to be well versed in not only the performance of open ablation procedures, but also in the various energy sources, lesion sets, endovascular options, hybrid open and endovascular options, and standalone thoracoscopic ablation techniques. Acquiring this knowledge often requires a focused externship at a high-volume center, along with strong partnership with your institutional electrophysiologists. This experience will not only allow for a healthy referral source, but also build inroads for collaboration in the pericardial approach to left atrial appendage closure devices and minimally invasive subxiphoid epicardial ablation procedures.

A knowledge base regarding energy-delivery devices and appendage-closure techniques is a key component to your success. Opportunities such as the James L. Cox Fellowship in Atrial Fibrillation Surgery offered by the AATS Graham Foundation offers a 90-day experience with 1 of 8 leaders in the field of anti-arrhythmia surgery. Additional courses such as the industry-sponsored Maze IV Surgical Training Program (AtriCure, Cincinnati, Ohio) are available for further education as well.

\section{END-STAGE HEART FAILURE SURGERY}

A field of continuing rapid development is that of surgical therapies for end-stage heart failure. Heart transplantation, temporary and permanent ventricular assist devices, and extracorporeal membrane oxygenator management are sources of high revenue for institutions; however, they require a lot of infrastructure and resources. An integral understanding among heart failure cardiologists, interventional cardiologists, and cardiac surgeons is key to establishing a heart failure/ cardiogenic shock center. Although this niche can be explored immediately after training in high-volume centers, some spend dedicated time to specialize in the perioperative selection and management of these patients.

The importance of available institutional resources, collaborating physicians, regional competition, and a financial model for the success of this program cannot be overstated. Societal memberships in The International Society for Heart and Lung Transplantation and the Heart Failure Society of America, among others, allow early exposure to colleagues and leaders in the field. A working knowledge of various bridging strategies to either permanent ventricular assist devices, or transplantation in combination with appropriate patient selection, is essential for personal and institutional success. Two of the current industry leaders in ventricular assist device technology-HeartWare International Inc 
(Framingham, Mass) and Thoratec Corporation (Pleasanton, Calif) — both offer fellowship courses in surgical heart failure therapy.

\section{A WORD ON LEADERSHIP}

To become a successful leader, you must first learn how to follow and how to be led. Even though you may be recruited into a leadership position of a program, leadership skills do not just come naturally to everyone and must be developed and perfected over time. To truly lead a program in your niche, you have to depend on your partners for backup, guidance in tough cases, navigation of the hospital climate, and mentorship. ${ }^{4}$ Ensuring that your chosen niche does not conflict with those of your partners is imperative, as is ensuring that your personal ambition will not jeopardize either your or your partners' programs.

Leadership is not merely dictating when and when not to perform a case, but all the administrative, interpersonal, and mentoring skills that are necessary in developing a program. The AATS Leadership Academy is a great way to get a broad view from current experts in the field of how to attain, cultivate, and groom leadership skills. Leadership does not generally come naturally for physicians, nor is it a checklist of things one acquires. Developing good leadership ability takes time, introspective reflection, and experience. Effective medical directorship combines a successful style of communication with coordination of people, for a common purpose, over a period of time. ${ }^{5}$

\section{BUILD A REPUTATION}

Once you have identified a niche and started to define your area, it is time for refinement and advertisement, which should occur in parallel with knowledge and experience aggregation. Success begets success, and advertisement in the form of article writing, lecturing, and attending conferences and presentations will begin to cement your area of expertise. Common advice for building a practice is that in your first 3 years you should become known at your own institution. In the next few years, the goal is to become known in a particular niche in your region. As your technical refinement and abilities progress, your goal is to be recognized nationally in a particular area.

Building a reputation takes effort and work. Whether in an academic setting or private practice, spending time with referring doctors, giving continuing medical education lectures, writing about your experiences, and getting involved in societies all take effort. However, a paramount element in this endeavor is to talk about your specialized area with everyone you can, to ensure that you are being recognized for your particular area. Even the shortest of papers or articles you write is worthwhile, to build your personal momentum as a thought leader in the field. At the end of a specified period of time, you want others to associate your name with the thought that "she/he does this type of surgery at this institution."

\section{CONCLUSIONS}

As important as building a niche is, many successful surgeons instead start in one arena and later convert to another, depending on their practice needs, interests, and the areas of growth in surgery. Developing your initial niche is one way of showcasing your expertise and cementing your role as a clinically competent surgeon. The temptation of newly graduated, surgical trainees is to think that they can take on anything. This trait is what gives us the perseverance to make it through the rigors of surgical training, but caution needs to be exercised in taking on challenges that cannot be conquered alone. The guidance of mentorship in choosing the appropriate niche at the appropriate place and time in transitioning to practice is essential to your success. Ensuring that you have good partners, departmental support, hospital resources, and appropriate supervision from mentors is key in avoiding failure.

Developing a niche and a reputation takes time. Most early career surgeons need to position themselves in an environment where they play a supportive role for a period of time, while being groomed to assemble a team and gain the confidence to lead a program. The steepest part of the learning curve for a surgeon is within the first 5 years of starting a surgical practice. An environment of surgical apprenticeship and supervised mentoring is key to ensuring a successful transition. Leadership needs to be practiced during this time, and a period of gradual responsibility is ideal, although it requires finding such an environment.

The 4 niches described here are all desirable, profitable, and add prestige to an institution, and as a result, they are high-profile positions and are heavily scrutinized. Realistic expectations regarding the complexity of the niche, the complexity of interpersonal leadership, as well as the time required to become an effective administrator, are necessary to ensure your success.

The future of adult cardiac surgery as a specialty is very exciting and bright. Perhaps more than at any other time in the development of the field, a specialized skillset and acquired experience are needed to push the boundaries and improve patient outcomes. Regardless of what area you ultimately choose as your niche, good surgeons with sound clinical judgment will always be needed.

\section{References}

1. Kron IL. Surgical mentorship. J Thorac Cardiovasc Surg. 2011;142:489-92.

2. Grondin SC. Building a successful career: Advice from leaders in thoracic surgery Thorac Surg Clin. 2011;21:395-415.

3. Nguyen TC, George I. Beyond the hammer: The future of cardiothoracic surgery J Thorac Cardiovasc Surg. 2015;149:675-7.

4. Tibayan F. " 10 things your mom forgot to tell you about the real world"-academic practice. Available at: http://www.ctsnet.org/sections/residents/featres articles/article-29. Accessed August 1, 2015.

5. Williams G, Wood EV, Ibram F. From medical doctor to medical director: Leadership style matters. Br J Hosp Med (London). 2015;76:420-2. 